\title{
Achieving Interoperability through Semantic Technologies in the Public Administration
}

\author{
Chiara Di Francescomarino, Mauro Dragoni, Matteo Gerosa, Chiara Ghidini, \\ Marco Rospocher, and Michele Trainotti
}

\author{
FBK-irst, Via Sommarive 18 Povo, I-38123, Trento, Italy \\ \{dfmchiara, dragoni, gerosa, ghidini, rospocher, mtrainotti\}@fbk. eu
}

\begin{abstract}
In this paper we report the experience of using semantic based tools and technologies for (collaboratively) modeling administrative procedures and their related documents, organizational roles, and services, in the Italian Public Administration (PA), focusing in particular on the interoperability aspects faced during the modelling process. This experience, the reported lessons learned and next steps identified, highlight the potential and criticality of using web 2.0 semantic technologies and tools to enhance participatory knowledge sharing, interoperability, and collaboration in the modeling of complex domains in the PA.
\end{abstract}

\section{Introduction}

In the last few years, the Public Administrations (PA) of several countries around the world have invested effort and resources into modernizing their services in order to improve labor productivity, as well as, PA efficiency and transparency. The recent contributions and developments in ICT (Information and Communication Technology) can boost this modernization process, as shown by the support the ICT can provide to the replacement of paper-based procedures with electronic-based ones (dematerialization of documents) within the PA. An important contribution of the ICT, in supporting the dematerialization of documents, is the production of proper and precise models of the administrative procedures of the PA and of the specific "entities" related to these procedures, such as the documents involved in the procedures, the organizational roles performing the activities, and the services needed to manage the electronic documents in an archival system. In fact, by following a model-driven approach [815], the availability of these models is a key factor towards both (1) the re-design and re-engineering of the administrative procedures, in order to replace paper-based documents with electronicbased ones, and (2) the definition of an appropriate archival system able to safely store, catalogue, manage, and retrieve the electronic documents produced within the PA. The definition of these models, which can act as "reference models" at the national level and enhance interoperability as described in [15], is often made complex, among the other problems, by the heterogeneity of procedures, document typologies, organizational structures, terminologies, and so on, present at regional or local level, due for instance to different regional laws or traditions.

In this paper, we report the experience of using semantic based technologies and a wiki-based modeling tool, MoKi [10], in the context of the ProDe Italian national project, in order to build national "reference models" for the management of electronic 
documentation in the Public Administration (PA). These models aim at representing both the domain entities and the processes in several fields of the PA with the focus on document management. Due to internal reasons the project didn't adopt available standard conceptual schemata for the representation of data and processes, but the different regional actors were initially asked to model their administrative procedures in a bottom up manner. This fact originated highly heterogeneous representations, which needed to be shared, reconciled, and eventually re-defined in terms of a common conceptual schemata subsequently adopted within the project, and in terms of common (pre-existing and ad-hoc) terminologies and meta-data. Here we highlight how MoKi was used to support participatory knowledge sharing and collaboration within the modeling activities of the different regions involved in the project in the spirit of the Web 2.0, and we report some lessons learned and future steps in our work, especially emphasizing the aspects related to the collaboration of PA employees of different regions, the reconciliation of local PA procedures into high level interoperable ones, the confluence of terminologies into shared lexicons, as well as the mapping between organizational and technological layers.

The contribution of the paper is twofold: first, it identifies the different interoperability aspects that originated in the context of the creation of reference models in a national project, it classifies them in light of standard interoperability models such as the "European Interoperability Framework for European public services" (EIF) [4], and it provides an overview of how MoKi and semantic based technologies have been used to face these issues. Second, it provides an attempt to report lessons learned and future steps especially related to how semantic-wiki based systems can support distributed modeling, the confluence of terminologies into shared lexicons, the adoption of (standard) pre-existing terminologies and metadata when available, and the integration of different entities for the construction of complex models. This contribution extends the work presented in [3] where the experience of the ProDe project was analyzed by looking at the usefulness of MoKi to support the collaboration process between knowledge engineers and domain experts in their modeling activities, and where aspects related to interoperability and construction or usage of shared knowledge were not considered.

The paper is structured as follows. In Section 2 we report related works concerning: (i) the usage of Semantic Web technologies in the PA domain, (ii) reference interoperability frameworks for the PA and (iii) model driven approaches towards interoperability. Section 3 provides an overview of ProDe and of the interoperability aspects in it. Section 4 describes how a semantic-based platform, based on the MoKi tool, has been implemented to face interoperability issues, while Section 5 presents how each interoperability aspect has been addressed within the ProDe project, what we learned from the experience and what we plan to do next. We conclude in Section 6 with some final remarks.

\section{Related Works}

Several works have focused on the application of Semantic Web technologies in the PA domain. We recall a few of them which have some commonalities with the work presented in this paper. In [17], the authors present a web-based knowledge management system that, by providing an up-to-date and accurate legal framework, supports (i) civil 
servants in the composition of administrative acts and (ii) civil servants, citizens and businesses in reasoning and substantiating administrative acts by means of precedents and opinions. In the context of the SAKE EU project, [19] proposes an ontology-based approach for the systematic management of changes in knowledge resources in public administrations. Successful applications of semantic wiki based technologies in the eGovernment domain have been reported in [1120], to favour the management and sharing of information and knowledge.

Some interoperability frameworks have been defined to grant the interoperability between different systems in the context of complex infrastructures. The Levels of Information Systems Interoperability (LISI) [1] initiative of the US Department of Defense aims to identify the stages through which systems should logically progress, or "mature", in order to improve their capabilities to interoperate. LISI considers five increasing levels of sophistication regarding system interaction and the ability of the system to exchange and share information and services. Each higher level represents a demonstrable increase in capabilities over the previous level of system-to-system interaction. A more recent framework, adopted by the European Commission, is the European Interoperability Framework for European public services (EIF) [4]. It defines a set of recommendations to support the delivery of European public services, by classifying the interoperability aspects to be addressed according to different interoperability levels (legal, organizational, semantic and technical).

Following the definition provided in [15], the approach taken in the ProDe project can be classified as a model-driven approach, where models have been systematically used as primary artifact for the definition of common procedures within different regions and for the engineering of the document management system. Of the three modeling sub-categories defined in [15], the models developed in ProDe cover the first and the second, that is, the specification of (domain) data - provided by means of OWL ontologies - and the specification of processes - provided by means of BPMN representations. The approach taken in ProDe, and the conceptual model developed to represent data, bring some relation with the effort carried put in the UK Government Common Information Model [13], where a reference model is defined to support the elicitation and setting out of the Requirements specifications for e-service development. Differently from [13], where the models are centered around the notion of e-service, the data models of ProDe are centered around the notion of document. Given the importance of documents within the project, data have been described in terms of the MoReq metadata standard [2], which in turn can be represented in terms of Dublin Core Metadata [5] as specified in [2]. Concerning the modeling of process knowledge, [15] classifies the efforts of the PA in two different families: (i) process modeling, and (ii) service modeling. In ProDe, the objective was to model general processes that are common to a large number of PA, and can therefore be classified as a process modeling effort. In that respect, the approach follows the one accomplished by SAP in the encoding of generic process models for different fields on its Solution Maps [16].

The ProDe project has been conceived keeping in mind the technological framework realized in ICAR 1 , a national project addressing the establishment of the Italian Public Connectivity and Cooperation System (SPC).

\footnotetext{
${ }^{1}$ http: //www.progettoicar.it/
} 


\section{Interoperability Aspects within ProDe}

ProDe ${ }^{2}$ is an Italian project with the aim of defining a national reference model for the management of electronic documentation (dematerialized document) in the Public Administration. This reference model follows an archival science perspective, and can be used for the identification of guidelines and functions needed to safely store, classify, manage, and retrieve, electronic documents produced within the PA in an archival system.

The project has a duration of 30 months (May 2010-October 2012) and the workplan is composed of 11 tasks assigned to 11 teams (task-teams) each one coming from one among 10 Italian regions. The 11 tasks are divided in 4 central tasks and 7 peripheral tasks. The 4 central tasks are in charge of guiding the activities and developing a common framework in which all the regions could recognize themselves. Each of the 7 peripheral tasks provides instead a specific expertise on a different sector of the PA and is in charge of guiding the modeling of administrative procedures for the sector it has been assigned to. Thus, the central tasks provide the main expertise in archival science, while the peripheral tasks provide domain expertise in different fields of the PA.

The setting and objectives of the project shows that interoperability aspects, of various nature and involving diverse actors and entities, play an important role within ProDe, In the following, we first briefly summarize the interoperability aspects as they naturally arose within the project, and then show how they relate to a standard interoperability framework such as EIF.

Users Interoperability. The development of a reference model for managing the dematerialization of documents demands the involvement of actors with different backgrounds, modeling skills, and responsibilities, spanning from experts in archival science, experts in laws, business process analysts, and knowledge engineers. Supporting the collaboration and cooperation among these actors to achieve the development of a shared reference model is even more crucial in ProDe, as (i) users from different regions distributed over Italy are required to contribute to the definition of such model, and (ii) the domains of the documents considered for dematerialization are various (e.g., healthcare, human resources, material resources, and so on).

Procedures Interoperability. The Italian legislation provides regions with a high degree of independence/freedom in writing new laws and in organizing their own structure to answer the citizens' needs. This explains why the 10 regions participating in the ProDe project: (i) have different levels of dematerialization; (ii) refer to different laws and regulations; (iii) use different methods, structures and terms for representing their administrative procedures. Nevertheless, as one of the goals of the project is to develop an archival system based on a common reference model shared by all the regions, this heterogeneity has to be taken into account in that the administrative procedures in place in the various regions, being understood their specificity, have to be compatible/compliant with the common conceptualization adopted.

Lexicon Interoperability. The freedom of Italian regions in self-organizing their structure and regulations is also reflected in the heterogeneity of the lexicon adopted in

\footnotetext{
${ }^{2}$ http: / / www .progettoprode.it/Home.aspx
} 
their administrative procedures. Indeed, it often happens that each region has its own name for designating documents reporting the same information, thus severely hindering comprehension of the process across regions.

Formal Language Interoperability. The management of document dematerialization requires to deal with different entities and artifacts: for instance, the (i) nature and properties of the documents to be dematerialized, (ii) the procedures and activities to store, catalogue, manage, and retrieve these document, and (iii) the actors involved in these activities. These entities have diverse intrinsic nature and are commonly formally represented with different modeling languages: for instance, documents and actors can be suitably modeled with declarative formalisms (e.g. ontologies), while business processes formalism are more appropriate to correctly represent procedures and activities.

Organizational and Technological Interoperability. In modeling the processes of a complex organization like a PA, it is common to identify at least two conceptual levels at which these processes take place: the organizational layer, comprising the activities, roles, processes, and organizational structure of the PA, and the technological layer, managing the set of information systems and software solutions that the PA uses to perform (part of) its activities. Although the conceptual connection between these two layers is rather evident, making it explicitly established and formalized in an integrated architecture enables to offer to complex organizations additional added-value services, like (i) verifying that the information systems supporting the organization are complaint with its processes, (ii) monitoring the execution of the organization processes, and (iii) checking (and possibly improve) the organization efficiency.

Placing ProDe Interoperabilities within the EIF. The interoperability issues encountered and identified in the ProDe project do not perfectly map to the four interoperability layers proposed by EIF[4]. This is mainly due to the different goals of the project and the framework: EIF is a set of recommendations that specify how European administrations should communicate with one another within the EU and across Member States borders in order to provide services; the ProDe project, instead, aims at defining national "reference models" of PAs' procedures and domain entities, starting from the existing local ones. This means that, for example, procedures carried out locally, are aligned at an abstract level, leaving regions the freedom to detail them according to their needs, so that the abstract version of a process model developed by a region can be used as base for the specificities of other regions.

Nevertheless, the interoperability aspects that came out within ProDe, are explicitly or implicitly related to the EIF interoperability layers. In detail:

- lexicon interoperability and formal language interoperability are related to the EIF SEMANTIC INTEROPERABILITY level. Both the interoperability aspects, in fact, deal with language heterogeneity that, hampering a common understanding, requires the provision of a "precise meaning" associated either to a shared vocabulary or to the relationships existing among different formal languages.

- procedures interoperability lies in the middle between the EIF LEGAL and ORGANIZATIONAL INTEROPERABILITY layers. The aspect deals, in fact, with the 
legislation and organization's procedure heterogeneity and the consequent need of their alignment, though at an abstract level.

- users interoperability relates to both the SEMANTIC and, at a higher level, the ORGANIZATIONAL INTEROPERABILITY layer. The interoperability among users with different backgrounds, competencies and roles, in fact, demands, on one hand, the achievement of a common understanding of their different views and, on the other, their "collaboration for the achievement of their mututally agreed goals".

- organizational and technological interoperability is orthogonal to the EIF layers: it in fact deals with the connection of the ORGANIZATIONAL and the TECHNICAL levels.

\section{Toward Achieving Interoperability in ProDe}

In order to face the interoperability issues described in the previous section, and to create a common reference model shared by all the regions belonging to the project, a common conceptual schema was proposed to the experts of the different task-teams to guide the modeling of their administrative procedures, the related documents, and the services to be provided by the document management system. This conceptual schema, whose simplified version is graphically depicted in Figure 1 using an Entity-Relationship notation, was developed by the experts in archival, computer, and organizational sciences working in the central tasks of the ProDe project, and it represents an extension of the one presented in $[3]^{3}$. In detail, the new entity Service is used to describe the functionalities required to the document management system by a given task in order to handle the documents managed within the task.

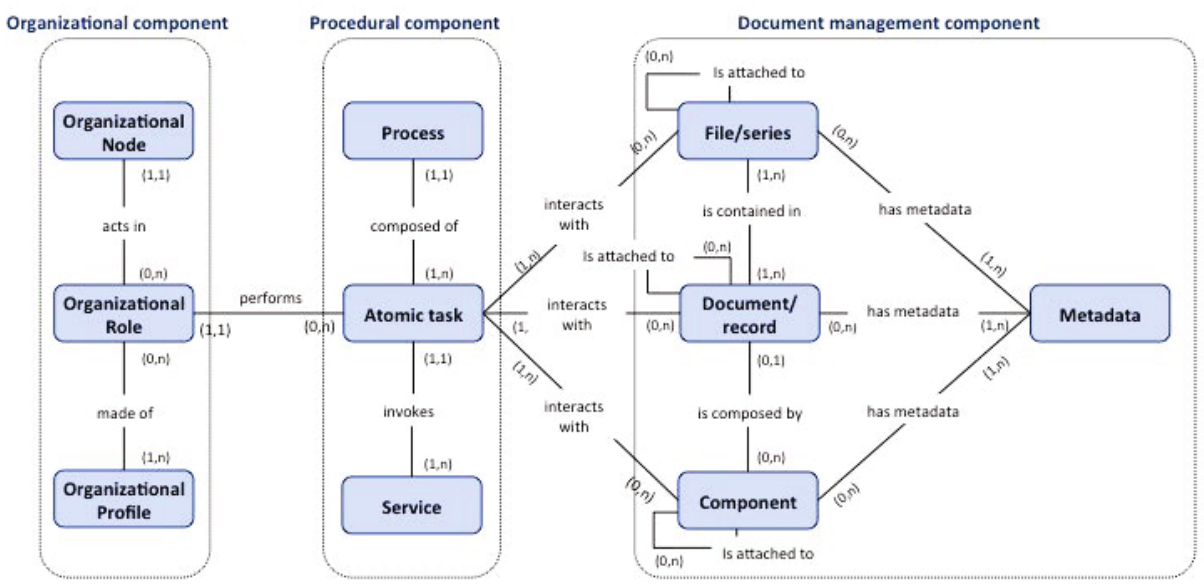

Fig. 1. The conceptual schema

\footnotetext{
${ }^{3}$ We omit an in depth description of the ProDe conceptual schema. The interested reader can refer to [3] for a detailed description.
} 
The second, and more important, contribution towards the achievement of interoperability was the customization and usage of a platform based on MoKi [9], a tool for collaborative modeling of integrated processes and ontologies, in order to obtain models following the conceptual schema presented in Figure 1. The platform developed for the ProDe project (hereafter referred to as the ProDeMoKi Platform) provides a set of MoKi installations: one installation for each of the peripheral tasks, hereafter named $P T_{1}, \ldots, P T_{7}$, and a single installation $C T$ for all the central tasks, where each MoKi installation $P T_{1}, \ldots, P T_{7}$ was connected with the one for the central task $C T$. The main idea of this platform is that, by using $C T$, the central tasks are able to create and manage entities (e.g., metadata for the description of documents) that are subsequently, and automatically, made available to $P T_{1}, \ldots, P T_{7}$ (e.g., to describe their documents), thus favoring convergence and re-use.

Next we show in detail the general architecture of the MoKi tool.

\subsection{The MoKi Architecture and Tool}

$\operatorname{MoK} 4$ [9] is a collaborative MediaWiki-based [12] tool for modeling ontological and procedural knowledge. The main idea behind MoKi is to associate a wiki page, containing both unstructured and structured information, to each entity of the ontology and process model. From a high level perspective, the main features of MoKi are:

- the capability to model different types of conceptual models, described in different formal languages, in an integrated manner. This feature is grounded on two different characteristics of MoKi. First of all, MoKi associates a wiki page to each concept, property, and individual in the ontology, and to each (complex or atomic) process in the process model. Special pages enable to visualize (edit) the ontology and process models organized according to the generalization and the aggregation/decomposition dimensions respectively. The ontological entities are described in Web Ontology Language (OWL [18]), while the process entities are described in Business Process Modeling Notation (BPMN [14]). Second, MoKi has extended the functionalities of the BPMN Oryx editor [6], to annotate process elements with concepts described in the ontology, or to incorporate data objects formalized in the ontology. The integrated procedural and ontological knowledge is then exported in a comprehensive OWL model following the approach described in [7].

- the capability to support on-line collaboration between members of the modeling team, including collaboration between domain experts and knowledge engineers. MoKi is an on-line tool based on MediaWiki, thus inheriting all the collaborative features provided by it. In addition MoKi facilitates the collaboration between domain experts and knowledge engineers by providing different access modes to the description (both structured and unstructured) of the elements contained in the model. In details, the current general version of MoKi is based on three different access modes:

\footnotetext{
${ }^{4}$ See also http: / /moki.fbk.eu

${ }^{5}$ The reader is referred to [10], where the architecture of MoKi has been presented in more details, by describing also how domain experts and knowledge engineers are able to exploit these different access modes in order to work collaboratively for modeling ontologies.
} 
Lightly-structured Access Mode: MO

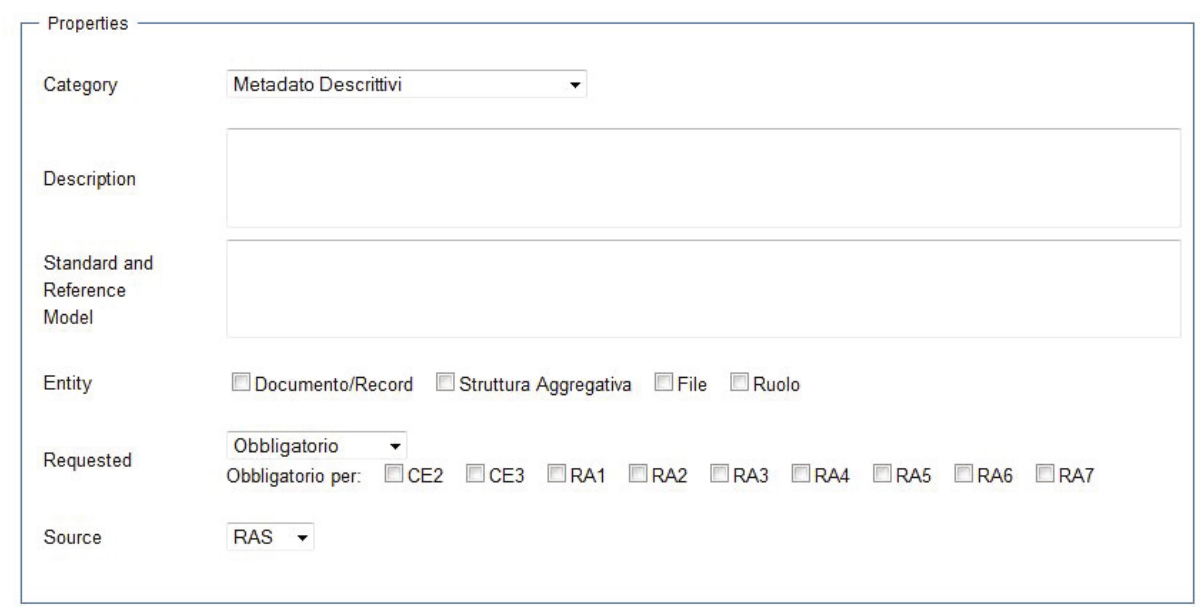

Save

Fig. 2. The template used to insert metadata information

- an unstructured access mode (for all users) to view/edit the unstructured content;

- a fully-structured access mode (for knowledge engineers) to view/edit the complete structured content; and

- a lightly-structured access mode (for domain experts) to view/edit (part of) the structured content in a simplified way, e.g. via light forms.

These features have been proved extremely important in the context of the ProDe project. In fact, the scenario addressed in the project required the modeling of administrative procedures, usually better described using a business process modeling notation, enriched with knowledge which typically resides in an ontology, such as the classification of document types, organizational roles, and so on. Moreover, the modeling team was composed by an heterogeneous group of domain experts and knowledge engineers situated in different Italian geographical regions.

Indeed, in the context of the ProDe project, many of the modeling actors involved in the ProDe project were not familiar with ontology modeling. Therefore, we facilitated the usage of MoKi by providing personalized lightly-structured access mode for each typology of entities that the users had to model (the ones in the Document management component, and Organizational structure component in Figure 1). An example of one of these personalized views is reported in Figure 2. The figure shows the template used for defining metadata entities.

Hereafter, we will refer to this version of MoKi providing personalized lightly-structured access mode as ProDeMoKi. 


\section{Interoperability in ProDe: What We Did, What We Learned and What We Will Do Next}

The ProDeMoKi Platform has been extensively used by 2 central task-teams and 6 peripheral task-teams 6 for the last 12 months. Overall, 2255 wiki pages have been created, 6809 revisions realized, 710 pages deleted and 71 pages renamed by both peripheral and central task users. Moreover, as comprehensively presented in [3], ProDeMoKi Platform users have been interviewed, by means of an on-line questionnaire, about the ease of use and the usefulness of the ProDeMoKi tool, in order to collect their subjective impressions.

The analysis of the huge amount of usage data (collected analyzing the MediaWiki database and the server log files), the users' subjective perception, and the concrete experience in the field gained during the project, allowed us to derive interesting observations about the support provided by semantic technologies to the different interoperability aspects demanded by ProDe (described in Section 3). In the following we report, for each of these interoperability aspects, the way in which it has been addressed by the ProDeMoKi Platform, the lessons we learned from the project experience and from the ProDeMoKi Platform usage, and some challenging ideas for future steps. Finally, we summarize some further related lessons learned.

\subsection{Users Interoperability}

The users involved into the ProDe project have different background based on their working area within the PA. Indeed, the domain experts, belonging to each of the peripheral task-teams, are specialized in specific topics, like healthcare, human resources, and financial resources. The MoKi platform provides a web-accessible knowledge sharing system that permits to all users - both within the same team and across different ones - to cooperate and to provide feedbacks about the modeled processes, and how documents are described in the platform.

Lessons Learned. In the evaluation of ProDeMoKi described in [3], we observed that about $45 \%$ of the users considered the collaboration support provided by ProDeMoKi one of the major strength of the tool and that users positively perceive its overall usefulness for the collaborative modeling of documents and processes. Such a usefulness is perceived more strongly by employees working in teams constituted by more than two persons (on average the usefulness of ProDeMoKi has been judged 3.8 out of a 5-point Likert scale for teams with more than two persons versus 2.8 for those with less than three). As presented in [3], there exists, in fact, a strong positive correlation between the size of the subject's team and his/her feedback about the ProDeMoKi usefulness for collaborative purposes. A similar relation (with the task-team's size) was also found for the perceived usefulness of the ProDeMoKi log history functionality. This functionality, although not frequently used by the peripheral task users (64 times in total), has been exercised $42 \%$ of times by the most productive ( 223 documents and 418 processes

\footnotetext{
${ }^{6}$ Two central and one peripheral task-teams are not required to use the ProDeMoKi Platform in this phase of the project.
} 
and tasks) and among the most numerous (4 modelers) task-teams, thus remarking its usefulness in case of large models and of collaborative work. These results show that the ProDeMoKi tool is particularly useful in situations in which users work in team on the same models.

Furthermore, the ProDeMoKi Platform is able to support the collaborative work of users with different backgrounds, by providing simplified views according to the roles and the specific competencies of the involved actors. Indeed, besides offering simplified views customized on the base of the specific domain (i.e., administrative procedures) to non-technical experts, the platform also tailors its interface to the specific actors' needs, e.g., offering different functionalities to the central and the peripheral task users. The extensive use of these views (the lightly-structured access mode of documents and the fully-structured access mode of processes have been accessed respectively 931 and 2533 times by the peripheral task users, while the central task-teams accessed the lightly-structured access mode of metadata and services 127 and 166 times, respectively) confirmed the usefulness of these simplified and customized views.

Next steps. We plan to better investigate with controlled experiments the collaboration mechanisms occurring among the different actors involved in the creation of interoperable models. The results and the feedback obtained will allow us to exploit the semantic web technologies to further support ProDeMoKi users in their modeling activities.

\subsection{Procedures Interoperability}

One of the aim of the ProDe project is to provide an archive of procedures representing the administrative processes of all the regions involved in the project. To this purpose, the ProDeMoKi Platform permits to all peripheral tasks to archive the process models they are in charge to deal with, and to make these models available to the users of the other peripheral and central tasks. This way, all users of the other tasks are able to verify the compliance between the processes stored in the archive and the ones actually used in their local government.

Lessons Learned. Differently from what happened in the modeling of document types and organizational aspects, where it was possible to identify relations and commonalities between the different models (taxonomies) produced by the different perhiperal task-teams already at the early stage of modeling, the formal representation of PA procedures generated a number of extremely different and heterogeneous process models. Such a variety and heterogeneity, due to granularity issues, different modeling styles, lack of guidelines and reference standards, and to the difference among regional procedures, hampered the convergence to the ProDe archive of procedures commonly agreed by all the participant regions. In this scenario the ProDeMoKi Platform enabled the identification of these diverging modeling styles from the very early stages of the project by allowing participatory knowledge sharing and fostering the communication and the discussion among regions, and held an crucial role in supporting the process of converging towards a uniform common model. Currently, the model contains 109 processes modeled by 6 peripheral task-teams (with an average of 18 processes per task-team). 


\subsection{Lexicon Interoperability}

The lexicon interoperability is one of the crucial issues of the ProDe project. As explained in Section 3, it is critical in order to avoid ambiguity problems that the domain experts are able to use a common lexicon for describing both the document properties and the atomic activities used in each process.

The effort spent during the project to this purpose was mainly devoted to: (i) the adoption of (standard) pre-existing terminologies and metadata (e.g. MoReq), when available; (ii) the creation of a shared vocabulary agreed among the different regions for the service definition.

The architecture of the ProDeMoKi Platform provides a mechanism to link the $C T$ installation and the $P T_{1}, \ldots, P T_{7}$ ones in order to grant the semantic interoperability of the used dictionaries, thus supporting regions in both these activities. Indeed, on one side, this linking functionality provides the tool with the capability to enable the definition of a common set of shared objects, that allowed the 4 central tasks to define a common set of metadata, services, functionalities and indicators to be used by all the peripheral tasks. On the other side, this functionality supports task-teams in reconciling synonyms and in mapping specific terms to more general and shared ones. This way, it allows them to come up with a common dictionary, based on MoReq, to be used by the peripheral tasks for describing both the document properties (metadata) and the services invoked by each atomic task.

Lessons Learned. The results of the effort spent on the convergence to a common dictionary, clearly appear in the reduction of the ambiguity of document and activity names in successive versions of the models created. For example, the number of different activities modeled dropped from more than 170, in the first version of the "Modello di riferimento", to 22 in the last version, with a relative reduction of about $87 \%$.

Next steps. The definition of high level models commonly agreed by all the participant regions, as well as the use of a shared set of metadata and of a common dictionary, represent the first step towards the possibility of (semi-)automatically verifying the compliance of the high level models to both national and regional laws, that is of primary importance for the PA. Moreover, the shared vocabulary of terms, fostering the definition of a mapping between the specific regional procedures and the commonly agreed models, could allow to verify the compliance of regional models to both national and local norms, and to support their adaptation to changes in the regulations.

\subsection{Formal Language Interoperability}

The complexity of PA procedures demands for the modeling and integration of different entities and artifacts. Each ProDeMoKi in the ProDeMoKi Platform permits to model the ontology of the documents, the processes in which they are used, and roles of the users involved in each process. To grant the interoperability of the formal languages used to describe these different conceptual models, the platform permits to build integrated models in which the entities defined in different formal languages can be semantically related, in order to better represent the PA procedures. An example is 


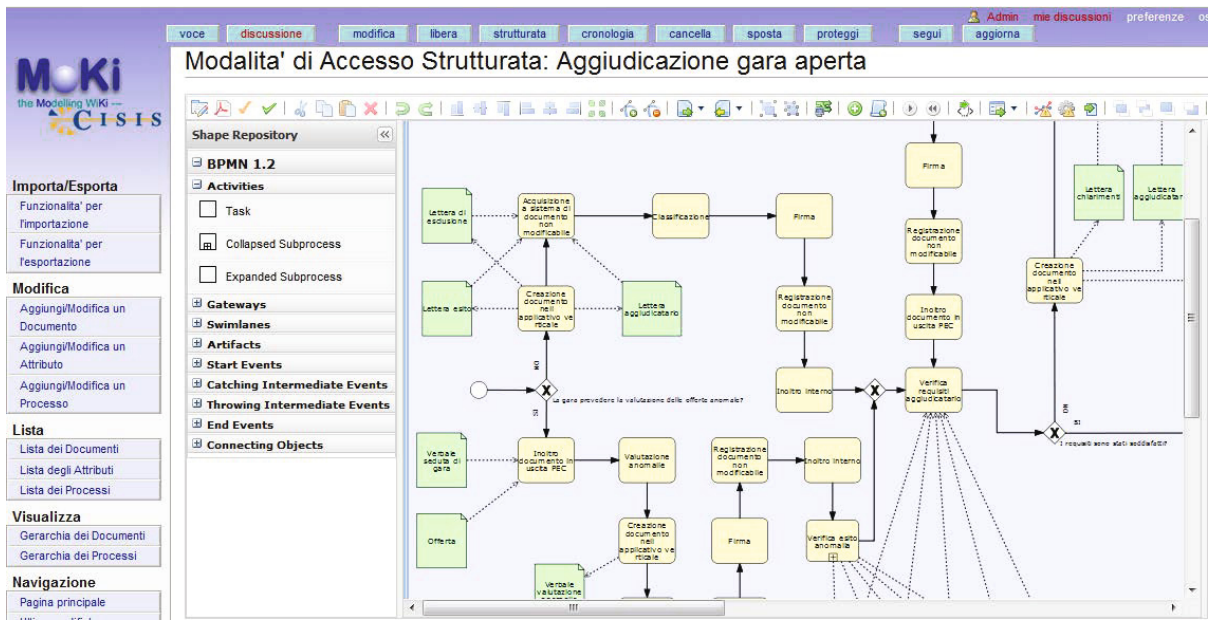

Fig. 3. The BPMN diagram enriched with documents

reported in Figure 3 in which the BPMN diagram shows how document entities are connected with processes.

Lessons Learned. The importance of the interoperability among the formal languages used for describing the different conceptual models of the PA procedures can be grasped also from the data related to the ProDeMoKi Platform usage. For example, 3.32 documents have been used, on average, in each process diagram, with peaks of about 20 different documents in a process (as shown in the boxplot in Figure 4a). Moreover, the same document has been used on average by 0.82 processes, including cases in which the same document has been used by $4 / 5$ different processes (Figure 4b). Users themselves are aware of the importance of such a facility: in fact, $45 \%$ of them judged such a capability of ProDeMoKi one of its major strengths.

\subsection{Organizational and Technological Interoperability}

As illustrated in Section 3, identifying and linking the organizational and the technological layers becomes a necessity when dealing with complex organizations like the PA, as occurred in the ProDe project.

Such an interoperability is achieved in the ProDeMoKi Platform thanks to the connection between each ProDeMoKi installation of the peripheral tasks with the one used in the central tasks. Indeed, as explained in Section 3, one of the goal of the central tasks is to define the services that can be invoked by each process modeled by the peripheral tasks. This way, the users of the peripheral tasks are able both to verify the completeness of the services provided, and to map these services with the organizational procedures and roles of their local government.

Lessons Learned. The conceptual difference between the organizational and technological layers is rather evident. However, we learned that the interoperability between 


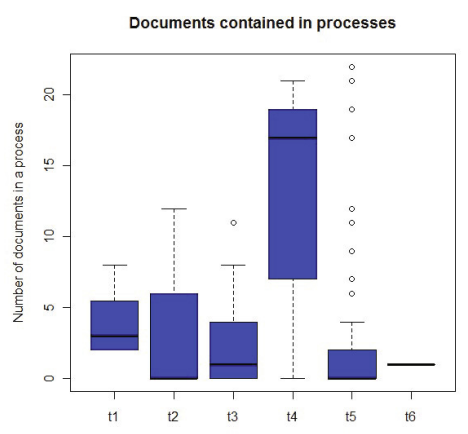

(a) Distribution of documents in processes per task-team

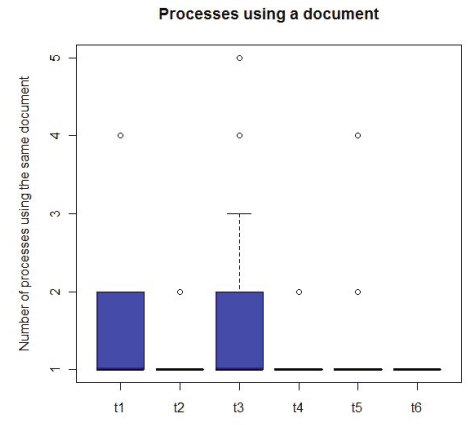

(b) Distribution of processes containing the same document per task-team

Fig. 4. Document and process integration

these two conceptual layers is hard to reach in the context of the ProDe project. This is mainly due to the several differences in the regions' organizations, that make the creation of a clear mapping between services, roles and procedures a challenging task.

Next steps. After having identified and modeled these two layers, the next step will be to draw formal relations between them. Being able to link these layers, for example determining when a certain technological component accomplishes a certain step in the business process, could allow us to monitor the PA organizational process by monitoring the progress of the corresponding process at the software layer. More importantly, when faced with a change in the organizational process we could automatically modify the technological process to reflect this change.

\subsection{Additional General Lesson Learned}

Among the peculiar findings of the project, we observed that PA employees are technological advanced users, even more than what we expected. Indeed, ProDeMoKi users not only work with a personal computer everyday for their job (mainly to write and read documents), but they are also people living in a world where the use of web technologies is constantly increasing. People navigate the Internet at home looking for news, events, restaurants; they use social networks to stay in touch with friends, online calendar to organize their lives and wikipedia when they want to research a specific topic.

Although PA employees don't commonly use Semantic Web technologies during their job, they use it everyday in their spare time. This is the reason why, for them, the use of a tool based on the same concept of the popular Wikipedia wasn't too challenging. We were surprised to discover that not only almost all the interviewed ProDeMoKi users frequently visit websites like wikipedia, but more than half of them edited at least one wiki page prior to use ProDeMoKi.

This familiarity with Semantic Web technologies allowed them to quickly learn how to use ProDeMoKi. Before the beginning of the modeling activities (February 2011), 
the PA employees have been trained with a learning session of 1 day, in which all the features of ProDeMoKi have been illustrated, and hands-on exercises proposed. After this session, the time spent for learning was very limited (on average, 1-2 days) and the learning process did not require the involvement of ProDeMoKi developers (the preferred approach was the autonomous training).

The proliferation of Semantic Web technologies, that we can envisage for a next future, could allow the quick and easy adoption of platforms like the ProDeMoKi Platform, as well as the growth of communities around these technologies. A further lesson we learned from the ProDe project, indeed, is the importance to actively involve users in the development process of the project, making them collaborating as part of a community. In the context of ProDe, the active participation and collaboration of PA employees of different regions, allowed to develop a common lexicon (by sharing knowledge and discussing), to refine models and procedures (by confronting them with those of other regions), and could allow, in the future, to keep alive the attention in maintaining and evolving the models built together as the PA procedures change.

\section{Conclusions}

The paper reports our experience in the construction and usage of solutions based on Semantic Web technologies in the context of ProDe, a national project involving Italian Public Administrations. In particular, it presents how these technologies enabled the collaborative modeling of administrative procedures and their related documents, organizational roles, and services, and contributed to deal with the interoperability issues emerged in the context of project. More specifically, the features provided by the MoKi tool and its customizations to face the specific needs of the project allowed to promote interoperability among: users, PA procedures, terminologies, conceptual models, and the different conceptual layers required by the project.

Taking advantage of the experience and of the lessons learned during the project, we plan, for the future, to better investigate and support the collaboration and interoperability mechanisms among users with different competencies and roles, as well as to explore techniques and approaches for (i) enabling the compliant evolution of PA procedures and laws; and (ii) monitoring the execution of PA procedures to check their compliance to models.

\section{References}

1. Levels of information systems interoperability (lisi) (1998), http: / /www . eng . auburn. edu/ hamilton/security / DODAF / LISI.pdf

2. MoReq2 specification: Model requirements for the management of electronic records (2008), http://ec.europa.eu/transparency/archival_policy/ moreq/doc/moreq2_spec.pdf

3. Casagni, C., Di Francescomarino, C., Dragoni, M., Fiorentini, L., Franci, L., Gerosa, M., Ghidini, C., Rizzoli, F., Rospocher, M., Rovella, A., Serafini, L., Sparaco, S., Tabarroni, A.: Wiki-Based Conceptual Modeling: An Experience with the Public Administration. In: Aroyo, L., Welty, C., Alani, H., Taylor, J., Bernstein, A., Kagal, L., Noy, N., Blomqvist, E. (eds.) ISWC 2011, Part II. LNCS, vol. 7032, pp. 17-32. Springer, Heidelberg (2011) 
4. European Commission. European Interoperability Framework (EIF) for European public services (2010), http://ec.europa.eu/isa/documents/isa_annex_ii_eif_en.pdf

5. DCMI. Dublin core metadata initiative (2007), http: / / dublincore.org/

6. Decker, G., Overdick, H., Weske, M.: Oryx - An Open Modeling Platform for the BPM Community. In: Dumas, M., Reichert, M., Shan, M.-C. (eds.) BPM 2008. LNCS, vol. 5240, pp. 382-385. Springer, Heidelberg (2008)

7. Di Francescomarino, C., Ghidini, C., Rospocher, M., Serafini, L., Tonella, P.: SemanticallyAided Business Process Modeling. In: Bernstein, A., Karger, D.R., Heath, T., Feigenbaum, L., Maynard, D., Motta, E., Thirunarayan, K. (eds.) ISWC 2009. LNCS, vol. 5823, pp. 114 129. Springer, Heidelberg (2009)

8. France, R., Rumpe, B.: Model-driven development of complex software: A research roadmap. In: 2007 Future of Software Engineering, FOSE 2007, pp. 37-54. IEEE Computer Society, Washington, DC (2007)

9. Ghidini, C., Rospocher, M., Serafini, L.: Moki: a wiki-based conceptual modeling tool. In: ISWC 2010 Posters \& Demonstrations Track: Collected Abstracts, Shanghai, China. CEUR Workshop Proceedings (CEUR-WS.org), vol. 658, pp. 77-80 (2010)

10. Ghidini, C., Rospocher, M., Serafini, L.: Conceptual modeling in wikis: a reference architecture and a tool. In: eKNOW 2012, The Fourth International Conference on Information, Process, and Knowledge Management, pp. 128-135 (2012)

11. Krabina, B.: A semantic wiki on cooperation in public administration in europe. Journal of Systemics, Cybernetics and Informatics 8, 42-45 (2010)

12. Wikimedia Foundation. Mediawiki, http://www.mediawiki.org

13. Cabinet Office Office of the e Envoy. e-services development framework primer v1.0b (2002), http: / / www.dcc.uchile.cl/ cgutierr/e-gov/eSDFprimer.pdf

14. OMG. BPMN, v1.1, www. omg.org/spec/BPMN/1.1/PDF

15. Peristeras, V., Tarabanis, K., Goudos, S.K.: Model-driven egovernment interoperability: A review of the state of the art. Computer Standards \& Interfaces 31(4), 613-628 (2009)

16. SAP. Solution maps, http: / / www1.sap.com/solutions/businessmaps/ solutionmaps/index.epx

17. Savvas, I., Bassiliades, N.: A process-oriented ontology-based knowledge management system for facilitating operational procedures in public administration. Expert Systems with Applications 36(3, pt. 1), 4467-4478 (2009)

18. Smith, M.K., Welty, C., McGuinness, D.L.: Owl web ontology language guide. W3C Recommendation, February 10 (2004)

19. Stojanovic, N., Apostolou, D., Ntioudis, S., Mentzas, G.: A semantics-based software framework for ensuring consistent access to up-to-date knowledge resources in public administrations. In: Metadata and Semantics, pp. 319-328. Springer, US (2009)

20. Wagner, C., Cheung, K.S.K., Ip, R.K.F., Bottcher, S.: Building semantic webs for egovernment with wiki technology. Electronic Government, an International Journal 3(1), 36-55 (2005) 